\title{
Ensuring quality at the ribosome
}

The mechanisms by which cells monitor the quality of proteins during translation are poorly understood. Work from the Weissman laboratory now identifies a ribosome-bound complex - which the authors name the ribosome quality control complex (RQC) - that degrades stalled proteins and signals stress to heat shock factor 1 (Hsf1).

The authors set out to further characterize the regulation and function of Hsf1, a conserved cytosolic transcription factor that coordinates protein quality control in eukaryotes. They first expressed a reporter for Hsf1 activity in a yeast full-genome library of loss-of-function alleles and found that Hsf1 activity was regulated by the loss of a range of genes. Notably, Hsf1 was strongly activated when the absence of particular genes (for example, those encoding chaperones and proteasomal components) resulted in increased protein misfolding. The authors then generated $\sim 78,000$ double mutants, looking for functional relationships between pairs of proteins that regulate $\mathrm{Hsf} 1$ activity.

They observed that the poorly characterized protein Ydr333c (which they named NPG/Vicky Summersby ribosome quality control $1(\mathrm{Rqc1})$ ) and the ribosome-bound E3 ligase listerin 1 (Ltn1) interacted with a similar set of translation-related proteins and so investigated them further.

First, to identify binding partners of Rqc1, this protein was immunoprecipitated and the precipitated complex (which the authors termed the RQC) was assessed by mass spectrometry. In addition to Rqc1, Ltn1 was found to be present in the RQC, along with the poorly characterized protein translation-associated element 2 (Tae2), the ATPase Cdc48 and its cofactors ubiquitin fusion degradation protein 1 (Ufd1) and nuclear protein localization protein $4(\mathrm{Npl} 4)$, and $~ 85 \%$ of the $60 \mathrm{~S}$ ribosomal subunits. Of note, Lys48-linked ubiquitin, which is a signal for protein degradation, was also present in the RQC, and Ltn1-mediated ubiquitylation was required for the recruitment of Cdc48 to this complex. Biochemical data and negative stain electron microscopy indicated that $\mathrm{Cdc} 48$ binds to the $60 \mathrm{~S}$ ribosome as part of the RQC.

So, what is the role of the RQC? Ltn 1 and Tae 2 are known to mediate the decay of proteins translated from mRNAs that lack a stop codon; translation of the mRNA poly(A) tail results in poly-Lys tracts (which is an example of a polybasic tract), and the nascent peptide chain is degraded by the proteasome. The authors designed a reporter containing a polybasic tract to assess whether the RQC has a role in co-translational degradation. Indeed, deletion of Rqc1, Tae2 or Ltn1 stabilized the polybasic tract reporter, suggesting that the RQC induces cotranslational degradation to promote quality control at the ribosome.

Previous studies suggest that the degradation of substrates containing polybasic tracts begins with the detection of stalled ribosomes. By assessing the degradation of the polybasic reporter and of endogenous polybasic stretches, the authors found that the RQC seems to target nascent peptide chains from stalled ribosomes for degradation. How the RQC recognizes stalled ribosomes is unclear, although data in this study indicate that Asc1 (an orthologue of the human scaffolding protein RACK1) and Hel2 (a ribosome-bound protein with a RING domain) act upstream of the RQC and thus might have a role in this process. Indeed, a role for Asc1 in co-translational quality control had previously been identified.

Finally, the authors looked back at the original connection that led them to discover the RQC - that the deletion of Rqc1 or Ltn1 induces Hsf1 activity. They found that deleting the RQC component Tae2 alone did not induce Hsf1 activity. As Hsf1 activity was not induced in yeast strains lacking both Tae2 and Rqc1, or Tae 2 and Ltn1, they conclude that Tae 2 is necessary to signal from the RQC to Hsf1, which leads to its activation under translational stress. Importantly, Tae 2 deletion did not prevent Hsf1 induction in strains mutated for genes encoding chaperones and proteasomal components, suggesting that translation stress activates Hsf1 independently of other stress pathways.

Thus, this study identifies a novel protein complex that signals translation stress to Hsfl. It will be interesting to see by which mechanism the RQC recognizes and binds stalled ribosomes.

Katharine H. Wrighton

ORIGINAL RESEARCH PAPER Brandman, O. et al. A ribosome-bound quality control complex triggers degradation of nascent peptides and signals translation stress. Cell 151, 1042-1054 (2012) 